\title{
Simulador computarizado de inmersión virtual como modelo de inicio de entrenamiento de laparoscopia urológica
}

\author{
Rodríguez-Covarrubias F, Martínez Liévano L, Gabilondo Pliego B, Gabilondo Navarro F, \\ Atisha-Fregoso Y, Arroyo C. \\ Departamento de Urología (FRC, BGP, FGN), Centro de Desarrollo de Destrezas Médicas (LML) y Departamento \\ de Reumatología (YAF) Instituto Nacional de Ciencias Médicas y Nutrición Salvador Zubirán. Departamento de \\ Cirugía. Hospital Universitario de Puebla (CA). Méjico.
}

Actas Urol Esp.2006;30(8):819-823

\section{RESUMEN}

SIMULADOR COMPUTARIZADO DE INMERSIÓN VIRTUAL COMO MODELO DE INICIO DE ENTRENAMIENTO DE LAPAROSCOPIA UROLÓGICA

Antecedentes: En la actualidad aún no está definido el mejor método de enseñanza para laparoscopia aplicada en urología, sin embargo se sabe que implica una larga curva de aprendizaje.

Métodos: Se llevó a cabo en nuestro Instituto un Curso de Laparoscopia Urológica que incluyó prácticas en un simulador computarizado de inmersión virtual en el que se evaluó las aptitudes de residentes que utilizaron rutinariamente el simulador como el grupo con experiencia (grupo 1) contra otro grupo sin experiencia (grupo 2). Se utilizaron diferentes modalidades en el simulador calificando la destreza y el tiempo para completar 4 tareas: coordinación, corte, colocación de clips y suturas endoscópicas.

Resultados: Las calificaciones promedio en las cuatro tareas fueron superiores en el grupo 1 comparado con el grupo 2, sin embargo nunca existió una diferencia estadísticamente significativa. Por otra parte en lo que corresponde al tiempo para realizar las prácticas fue menor en el grupo 1 comparado con el grupo 2, con diferencia estadísticamente significativa.

Conclusiones: El desempeño de los alumnos sin experiencia en el uso de simuladores fue similar al de los alumnos habituados a este tipo de entrenamiento, sin embargo, se observó una mayor velocidad en la realización de las tareas conforme se aumenta el tiempo del uso de estos simuladores. El uso de simuladores permite el aprendizaje de técnicas básicas permitiendo mejorar la destreza y coordinación para la práctica de laparoscopia.

Palabras clave: Laparoscopia urológica. Simuladores virtuales. Cirugia de mínima invasión.

\section{ABSTRACT \\ USE OF A VIRTUAL INMERSION COMPUTER SIMULATOR AS A MODEL FOR BASIC TRAINING IN LAPAROSCOPIC UROLOGY}

Background: to date, it has not been defined the best method for teaching urologic laparoscopy, however it is well recognized that it involves a steep learning curve.

Methods: A course of Laparoscopic Urology was done in our Institute. The program included skill practices in a virtual immersion simulator which evaluated, the score and time to complete each activity. This was done in a group of residents with previous experience with this virtual simulator (group 1) and another group of residents with no experience (group 2). Four different basic tasks were performed in the virtual simulator, which included: coordination, cutting, clip application and performing a simple suture.

Results: When we compared the scores between both groups the mean scores for each task were superior in group 1 compared to the group 2 , with no statistically significant difference, however when we compared the time to complete each task, it was shorter in group 1 compared to group 2 with a statistically significant difference.

Conclusions: The performance of residents without experience in a virtual simulator was similar to that of previously trained residents, however it takes less time to complete each task as the resident gains experience in these simulators. The use of virtual simulators for laparoscopy training are useful when learning basic techniques allowing the surgeon to improve hand dexterity and coordination in laparoscopic surgery.

Keywords: Urologic laparoscopy. Virtual simulators. Minimal invasive surgery. 
$\mathrm{D}$ esde el primer caso del uso de un abordaje de invasión mínima en urología se planteó el alto grado de dificultad de esta técnica ${ }^{1}$. Actualmente los procedimientos quirúrgicos y el número de casos que se realizan por un abordaje laparoscópico continúa aumentándose. En la actualidad, sus indicaciones se han expandido hasta abarcar la mayoría de las cirugías urológicas, incluyendo procedimientos oncológicos y reconstructivos complejos $^{2-3}$, sin embargo, en nuestro medio, el número de especialistas en urología con entrenamiento en cirugía de invasión mínima es escaso para el número de casos que podrían ser resueltos por este abordaje. Existen varias limitaciones, entre las que se encuentran la dificultad técnica de este abordaje con una curva de aprendizaje muy larga, aunado a que no se ha encontrado el método ideal para la enseñanza de esta cirugía. Entre las modalidades de entrenamiento utilizadas alrededor del mundo están: residencias, cursos de sub-especialización, instrucción tutorial intraoperatoria, cursos con prácticas en modelos animales, uso de 'entrenadores' pélvicos y, más recientemente, el uso de simuladores virtuales, que surgen a partir de los simuladores de vuelo que utilizan los pilotos de avión para su entrenamiento.

Sin duda, la mejor opción parece ser el entrenamiento tutorial intraoperatorio, sin embargo, el tiempo quirúrgico prolongado y el riesgo de complicaciones hacen surgir cuestionamientos éticos y financieros sobre la eficacia de este método ${ }^{4}$. Actualmente, la forma más segura y eficaz para iniciar el entrenamiento en cirugía laparoscópica es el uso de simuladores virtuales ${ }^{5}$. En condiciones de tiempo real, las prácticas en este tipo de simuladores pueden adaptarse de acuerdo a las necesidades y metas de cada alumno, sin presiones externas y con toda seguridad, con la ventaja de que las rutinas pueden repetirse cuantas veces se considere necesario para acostumbrarse al equipo y las actividades ${ }^{6}$; sin necesidad de utilizar animales de experimentación con fines de entrenamiento $^{6}$. En la actualidad existen artículos de simuladores para la resección transuretral de próstata $^{7}$ y ureteroscopia ${ }^{8}$, pero no existen casos sobre su aplicación en urología laparoscópica. Aportamos nuestra experiencia en la enseñanza de técnicas de invasión mínima mediante un simulador de inmersión virtual a un grupo de residentes de urología.

\section{MATERIAL Y MÉTODOS}

En el mes de agosto del 2005, se llevó a cabo el Primer Curso Teórico-Práctico "Dr. Jorge Elías Dib" de laparoscopia en urología ${ }^{9}$ en las instalaciones del Centro de Desarrollo de Destrezas Médicas (CEDDEM) del Instituto Nacional de Ciencias Médicas y Nutrición Salvador Zubirán con asistencia de 83 personas, entre las cuales se incluyeron 20 residentes de urología. La duración del curso fue de 72 horas, tiempo en el que se impartieron 30 conferencias sobre laparoscopia básica y avanzada aplicada a la urología con una duración de 15 horas de teoría. En el turno vespertino, con duración de 12 horas, se realizaron prácticas en forma rotatoria entre los asistentes de: nefrectomía laparoscópica en modelo animal canino, suturas endoscópicas en "entrenador pélvico” con cámara laparoscópica y prácticas en simulador computarizado de inmersión virtual de laparoscopia básica (LapSim, Immersion Medical, software Surgical Science versión 3.01 con el módulo 'basic skills', Goteborg, Suecia).

Durante este curso se realizó un estudio prospectivo, comparativo, abierto en el que se evaluó a los residentes del Instituto que tienen acceso ilimitado al simulador, comparados con los asistentes sin experiencia en el uso del simulador, cuando utilizaron el simulador de inmersión virtual durante el curso, sin saber que estaban siendo evaluados. La computadora calificó la destreza manual y el tiempo para realizar 4 rutinas básicas de laparoscopia que incluyeron: coordinación, corte, aplicación de clips y realización de suturas endoscópicas (Fig. 1). Todas estas fueron calificadas de 0 a 100 por el software del simulador así como el tiempo que emplearon para completar la actividad o fueran reprobados por la computadora. Estos resultados se analizaron en forma individual y grupal utilizando la prueba $\mathrm{U}$ de Mann-Whitney.

\section{RESULTADOS}

Durante el curso de laparoscopia en urología, se evaluó de forma prospectiva a 20 residentes de urología de distintos centros hospitalarios en México que asistieron al curso (grupo 2), que se 

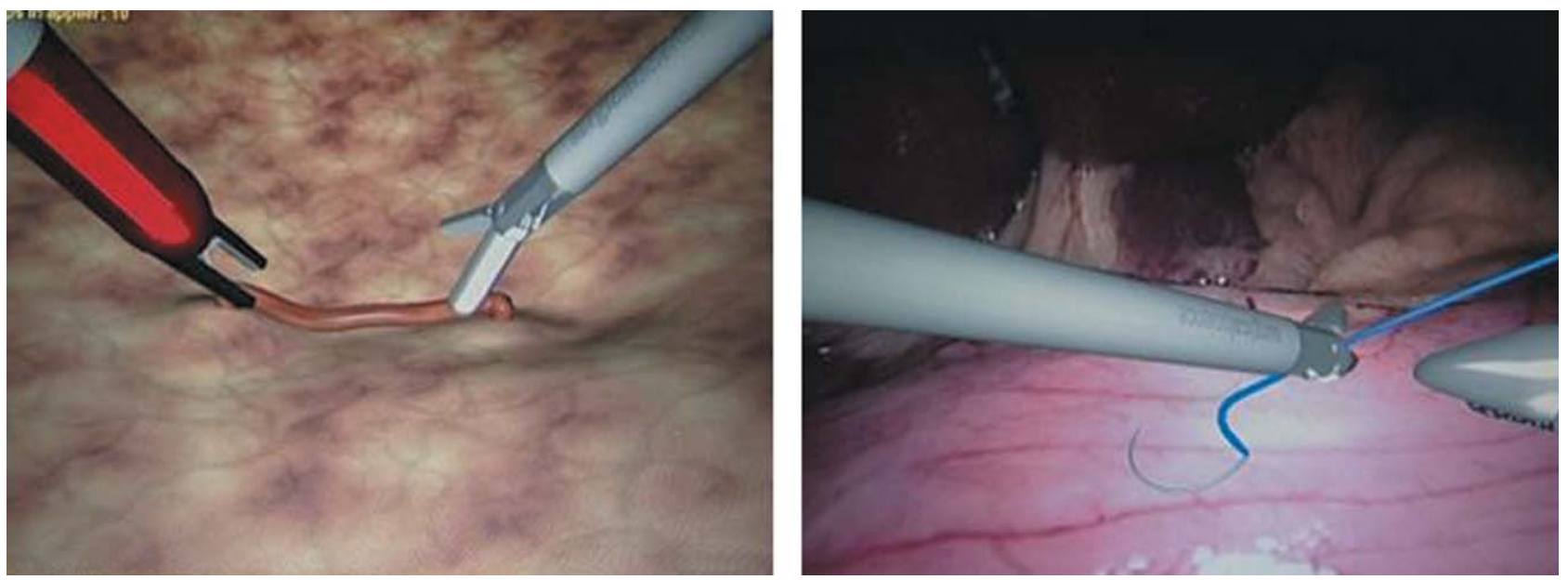

FIGURA 1: Se demuestra la imagen del simulador en las rutinas de corte y aplicación de clips en el recuadro izquierdo, y en la práctica de aplicación de sutura en el recuadro derecho.

compararon con las calificaciones obtenidas por residentes del Instituto que tienen un mayor acceso al simulador y mayor experiencia en su uso (Grupo 1). Se analizó el desempeño en el simulador de inmersión virtual de 14 de ellos. Se comparó la destreza de 4 residentes en el grupo 1 con experiencia en el uso del simulador (12 horas en promedio, rango de 4 a 19 horas) con la destreza adquirida durante el curso de 10 residentes sin experiencia previa (grupo 2), con un tiempo limitado en el uso del simulador (73,3 minutos en promedio, rango de 22 a 162 minutos).

La primera tarea asignada en las prácticas fue la de coordinación mano-ojo. Los alumnos del grupo 1 obtuvieron una calificación promedio de 74,5 y los del grupo 2 de 70,4, sin diferencia estadísticamente significativa $(\mathrm{p}=0,52)$. El promedio de tiempo para completar esta práctica fue de 57 segundos para el grupo 1, comparado con 79 segundos en promedio para el grupo 2 con una diferencia significativa $(p=0,03)$. En la práctica de corte, los alumnos del grupo 1 obtuvieron una calificación promedio de 85,2 y los del grupo 2 de 77,9 , sin diferencia estadísticamente significativa $(\mathrm{p}=0,35)$, sin embargo, el tiempo promedio fue menor para el grupo 1 (89 contra 142 segundos respectivamente) con una diferencia significativa $(\mathrm{p}=0,04)$. En la tercera tarea de aplicación de clips, la calificación promedio para el grupo 1 fue de 79,6 y para el grupo 2 de 78,2 sin diferencia estadísticamente significativa $(\mathrm{p}=0,85)$, en lo que corresponde al tiempo para desarrollar la tarea fue ligeramente menor en el grupo 1 (95 contra 143 segundos respectivamente), con una tendencia estadística $(\mathrm{p}=0,06)$. Finalmente, al realizar la práctica de la sutura endoscópica el grupo 1 obtuvo una calificación promedio de 79,8 y el grupo 2 de 77,9 sin diferencia estadística $(p=0,43)$ y en esta actividad el tiempo promedio fue similar entre ambos grupos (385 contra 425 segundos respectivamente), sin ser estadísticamente significativa la diferencia $(\mathrm{p}=0,43)$. (Los resultados se resumen en la Tabla 1).

\section{DISCUSIÓN}

Desde que en 1991, se introdujo la cirugía laparoscópica aplicada a la urología $^{1}$, su utilidad se ha expandido en forma importante, y la demanda de cursos de entrenamiento en laparoscopia básica y avanzada se ha incrementado en forma paralela. Sin embargo, aún no se ha definido el método óptimo para la enseñanza de estas técnicas. Actualmente, el entrenamiento en laparoscopia incluye una fase básica y una fase clínica. La fase básica involucra prácticas en laboratorio, y generalmente se lleva a cabo con "entrenadores pélvicos", cirugía en modelos animales y disección en cadáveres ${ }^{10}$.

Recientemente se ha descrito la utilidad de simuladores computarizados de inmersión virtual similares a los que se utilizan para el entrenamiento de pilotos de avión para el entrenamiento en técnicas básicas de laparoscopia. Su uso tiene como ventaja la práctica continua en 
Tabla 1

Se enumeran por grupos la calificación otorgada por el simulador para cada una de las actividades, así como el tiempo empleado para completarlas

\begin{tabular}{lcccc}
\hline Actividad & \multicolumn{2}{c}{ Grupo I } & \multicolumn{2}{c}{ Grupo II } \\
& Calificación & $\begin{array}{c}\text { Tiempo } \\
\text { segundos }\end{array}$ & Calificación & $\begin{array}{c}\text { Tiempo } \\
\text { segundos }\end{array}$ \\
\hline Coordinación & 74,5 & $57^{*}$ & 70,4 & $79^{*}$ \\
Corte & 85,2 & $89^{*}$ & 77,9 & $142^{*}$ \\
Clip & 77,6 & $95^{*}$ & 78,2 & $143^{*}$ \\
Sutura & 79,7 & 385 & 77,9 & 425 \\
\hline
\end{tabular}

$* \mathrm{p}<0,05$

tiempo real, lo cual permite una medición objetiva de las habilidades y destrezas adquiridas, tales como exactitud y precisión aplicada durante el ejercicio, con la consecuente retroalimentación del progreso en las diferentes actividades para el usuario ${ }^{11}$. Por otra parte, debido a que el ambiente de práctica es seguro y libre de complicaciones, el alumno tiene la oportunidad de fallar y aprender de dichos errores, lo cual es prácticamente imposible en un medio puramente clíni$\mathrm{co}^{12}$. Sus inconvenientes incluyen el alto costo de adquisición y mantenimiento, la ausencia de retroalimentación táctil (similar a lo que se encuentra en cirugía robótica sin la posibilidad de la tercera dimensión con la que se cuenta en equipos como el DaVinci ${ }^{\mathrm{TM}}$ ) y el uso de algoritmos generados por computadora para representar la interacción de los tejidos con el instrumental, por lo cual la fidelidad de las imágenes es crucial ${ }^{11}$. Hyltander et al. realizaron un estudio con 24 estudiantes de medicina y los asignaron en forma aleatoria a un grupo de entrenamiento con LapSim o a un grupo control sin entrenamiento. La evaluación final comprendió la realización de rutinas similares a las desarrolladas en el simulador virtual pero en un modelo porcino. El grupo con entrenamiento tuvo un mejor desempeño en todas las tareas asignadas en el laboratorio, en comparación con el grupo sin entrenamiento, tanto en velocidad como en destreza. Este estudio sugiere que las habilidades obtenidas en el simulador virtual LapSim pueden ser transferidas a la práctica in vivo ${ }^{13}$. En nuestro caso, los alumnos con experiencia previa en el uso del simulador virtual, obtuvieron calificaciones mayores en las 4 prácticas con menor tiempo requerido para completarlas. Sin embargo, no se encontró diferencia estadísticamente significativa en el promedio de calificaciones de ambos grupos, lo cual posiblemente demuestra la rapidez con la que se puede obtener destreza mediante el uso de simuladores virtuales o el interés que tienen los residentes para aprender estas técnicas. Lo que si se demostró, es que el uso del simulador por el grupo entrenado les permitió realizar las mismas rutinas en menor tiempo, (excepto en la aplicación de suturas que podría explicarse por el alto grado de dificultad de esta aptitud) que tiene un beneficio en la práctica clínica, puesto que podría impactar en el tiempo quirúrgico y complicaciones de un procedimiento prolongado, sin embargo, está pendiente un estudio comparativo de ensayos en modelos animales para verificar lo anterior.

Una limitación de nuestro estudio, es el reducido número de participantes, pero consideramos que la posibilidad de reunir dos grupos con nuestras características lo hacen un estudio inicial útil. Por otra parte, será importante medir la destreza adquirida en el simulador mediante prácticas en modelos animales o cadavéricos y, además, comparar este método de aprendizaje con otros ya establecidos, ya que algunos autores sugieren que el uso de modelos cadavéricos para el entrenamiento en laparoscopia proporciona un ambiente quirúrgico ideal que permite realizar disecciones que asemejan a los procedimientos urológicos reales con la ventaja de que no hay sangrados activos durante las prácticas que disminuye el nivel de estrés de los asistentes ${ }^{14}$.

El advenimiento de la cirugía laparoscópica como una modalidad estándar de tratamiento en urología, junto con las exigencias de aprendizaje para los especialistas, ha hecho que parte de la investigación se enfoque en perfeccionar los métodos de enseñanza de las técnicas de invasión mínima. Pese a las desventajas que puedan ofrecer los simuladores virtuales, en nuestra opinión, son una opción útil y viable para iniciar el entrenamiento de residentes y especialistas interesados en obtener habilidad en laparoscopia, 
sobre todo en nuestro medio, en el que los programas de residencia aún no incluyen en forma rutinaria la práctica de la cirugía laparoscópica.

\section{CONCLUSIÓN}

Las indicaciones para realizar procedimientos por vía laparoscópica en urología se han incrementado en forma dramática en la última década. Esto se traduce en la necesidad de un número mayor de urólogos con entrenamiento en cirugía de invasión mínima. Hasta ahora no se ha definido cuál es el mejor método de aprendizaje, $\mathrm{y}$ esto se refleja en nuestro medio, como en muchos otros países, en los que los programas de residencia aún no cuentan con enseñanza rutinaria de dicho abordaje. Los simuladores computarizados de inmersión virtual ofrecen la posibilidad de adquisición de destreza básica para la práctica de laparoscopia, y proporcionan un ambiente seguro para el entrenamiento, con la posibilidad de la medición objetiva de aciertos y errores, que se traduce en un mayor aprendizaje y retroalimentación continua para el alumno.

\section{REFERENCIAS}

1. Clayman RV, Kavoussi LR, Soper NJ, Dierks SM, Meretyk S, Darcy MD, et al. Laparoscopic nephrectomy: initial case report. J Urol. 1991 Aug; 146(2):278-282.

2. Katz R, Nadu A, Olsson LE, Hoznek A, de la Taille A, Salomon L, et al. A simplified 5-step model for training laparoscopic urethrovesical anastomosis. J Urol. 2003 Jun; 169 (6):2041-2044.

3. Rassweiler J, Frede T, Guillonneau B. Advanced Laparoscopy. Eur Urol. 2002;42(4): Curric Urol 1-12.

4. Traxer O, Gettman MT, Napper CA, Scott DJ, Jones DB, Roehrborn CG, et al. The impact of intense laparoscopic skills training on the operative performance of urology residents. J Urol. 2001 Nov;166(5):1658-1661.
5. Fichera A, Prachand V, Kives S, Levine R, Hasson H. Physical reality simulation for training of laparoscopists in the 21st century. A multispecialty, multi-institutional study. JSLS. 2005 Apr-Jun;9(2):125-129.

6. Hackethal A, Immenroth M, Burger T. Evaluation of target scores and benchmarks for the traversal task scenario of the minimally invasive surgical trainer-virtual reality (MIST-VR) laparoscopy simulator. Surg Endosc. 2006 Apr; 20(4):645-650.

7. Kallstrom R, Hjertberg H, Kjolhede H, Svanvik J. Use of a virtual reality, real-time, simulation model for the training of urologists in transurethral resection of the prostate. Scand J Urol Nephrol. 2005;39(4):313-320.

8. Knoll T, Trojan L, Haecker A, Alken P, Michel MS. Validation of computer-based training in ureterorenoscopy. BJU Int. 2005 Jun;95(9): 1276-1279.

9. Arroyo C, Elias Dib J. Primer curso teórico-práctico de laparoscopia en urología. Rev Mex Urol. 2005;65(6):480-481.

10. Shalhav AL, Dabagia MD, Wagner TT, Koch MO, Lingeman JE. Training postgraduate urologists in laparoscopic surgery: the current challenge. J Urol. 2002 May; 167(5):21352137.

11. Feldman LS, Sherman V, Fried GM. Using simulators to assess laparoscopic competence: ready for widespread use? Surgery. 2004 Jan; 135(1):28-42.

12. Kneebone R. Simulation in surgical training: educational issues and practical implications. Med Educ. 2003 Mar;37 (3):267-277.

13. Hyltander A, Liljegren E, Rhodin PH, Lonroth H. The transfer of basic skills learned in a laparoscopic simulator to the operating room. Surg Endosc. 2002 Sep;16(9):1324-1328.

14. Katz R, Hoznek A, Antiphon P, Van Velthoven R, Delmas V, Abbou CC. Cadaveric versus porcine models in urological laparoscopic training. Urol Int. 2003;71(3):310-315.

Dr. C. Arroyo

Departamento de Cirugía

Hospital Universitario de Puebla

13 sur y 25 poniente, Col Volcanes

Puebla, México

e-mail: jcak1@yahoo.com

(Trabajo recibido el 19 de diciembre 2005) 